\title{
EXTREME NORMALIZED ANALYTIC FUNCTIONS WITH POSITIVE REAL PART
}

\author{
MAURICE HEINS
}

1. Let $P$ denote the set of analytic functions $f$ on a given Riemann surface $S$ which have positive real part and are normalized to satisfy the condition $f(a)=1$, where $a$ is a given point of $S$. The family $P$ is convex and compact. The existence of extreme points of $P$ is assured by the Krein-Milman theorem - cf. $[9,130]-$ or in a more elementary fashion by a proof using the argument of my paper [7].

We remark that the "Schwarz lemma" extremal problem for $(S, a, b)$ where $b(\neq a) \in S$, namely: "What are the $g$ analytic on $S$, satisfying $|g|<1, g(a)=0$, which maximize $|g(b)|$ ?" has in any case solutions that are of the form of the composition of a Möbius transformation $m$ mapping $\{\operatorname{Re} z>0\}$ onto $\{|z|<1\}, m(1)=0$, with an extreme point of $P$. We observe concomitantly that there exist $S \notin O_{A B}$ such that no extreme member of any associated $P$ has as real part a singular positive harmonic function or a finite sum of minimal positive harmonic functions. The assertion may be established with aid of the method of the celebrated construction of P. J. Myrberg [10].

We are led to the conclusions that without finiteness conditions on the topological structure of $S$ (relative to the genus and the number of boundary elements in the sense of Kerékjártó and Stoilow) the extreme members of $P$ may have real parts with quite complicated structure and that the simplicity of the "Schwarz lemma" extremals present in the case where $S$ has finite topological characteristics and nonpointlike boundary components (for which cf. [1], [4], [5]) does not persist.

The object of the present paper is to characterize the extreme members of $P$ for the cited "reasonable" situation where $S$ has finite topological characteristics and nonpointlike boundary components (Theorem 5.1). Forelli [3] showed that in the important subcase where $S$ is a plane region of connectivity $n$ the extreme functions are exactly the members of $P$ having constant valence $n$ on $\{\operatorname{Re} z>0\}$. Accounts of such maps are to be found in [2] — modulo a Möbius transformation - and [6]. We shall give an account (Section 3) of the plane case following the formulation of Grunsky in [6], which brings to the fore a natural correspondence between the extreme functions and the cartesian product of the boundary components when $S$ is taken to have a "reasonable" boundary. Valuable historical notes are given in [6]; cf. p. 198. We remark that maps generically of this kind were envisaged by Riemann in the last section (Section 22) of his Dissertation [11, 42-3] and in [11, 440-4]. 
For the case where $S$ has finite positive genus $g$ and $c$ boundary components Forelli [3] showed that the extreme functions are contained in the subset of $P$ whose members have constant valence on $\{\operatorname{Re} z>0\}$, the value of the valence belonging to $\{c, \ldots, 2 g+c\}$, and that if a member of $P$ has constant valence $c$ on $\quad\{\operatorname{Re} z>0\}$, it is an extreme function.

In the present paper we characterize the extreme functions in terms of the period vectors of the normalized minimal positive harmonic functions on $S$. Cf. [8].

The study of the real part of an extreme function, which is basic, is treated by Forelli with the aid of Poisson type representations derived by uniformization methods. Here the study is based on elementary extremal considerations concerning positive harmonic functions and classical results concerning sub- and superharmonic functions (Lemma 3.2).

We plan to apply the results of this paper elsewhere to finite Pick-Nevanlinna problems.

2. The case: $S=\{|z|<1\}, a=0$. Here the situation is prototypical for the plane theory. The extreme functions are given by

$$
f_{\eta}: z \mapsto(\eta+z) /(\eta-z),|z|<1,
$$

where $|\eta|=1$. That each $f_{\eta}$ is extreme follows on direct examination of the terms entering a proposed representation as a barycenter of two members of $P$ with particular reference to their behavior at points of $\{|z|=1\}$. If $f$ is an extreme point of $P$, then $\lim _{\eta} \operatorname{Re} f=0,|\eta|=1$, save for exactly one $\eta$, say $\zeta$, as we see with the aid of the Poisson-Stieltjes integral representation of $f$. We conclude that $f=f_{\zeta}$.

3. The case where $S$ is a plane region of finite connectivity $n+1(>1)$. We suppose, as we may, that $S$ is a bounded region $\Omega \subset C$, the frontier of which consists of $n+1$ disjoint regular analytic Jordan curves $\Gamma_{1}, \ldots, \Gamma_{n+1}$, the component $\Gamma_{n+1}$ separating $\Omega$ from $\infty$.

Given $u$ real-valued harmonic on $\Omega$, we introduce the period vector $\omega(u)=$ $\left(\omega_{1}(u), \ldots, \omega_{n}(u)\right)$, where $\omega_{k}(u)$ is the period of the conjugate of $u$ along a level line of the harmonic measure of $\Gamma_{k}$ with respect to $\Omega$, the level being less than but near 1 and the sensing being that given by homotopic deformation from $\Gamma_{k}$ positively sensed.

Suppose that for $k=1, \ldots, n$ the function $u_{k}$ is positive harmonic on $\Omega$ and vanishes continuously on $\Gamma-\Gamma_{k}$. Then $\omega_{j}\left(u_{k}\right)<0, j \neq k$. By Green's theorem $\omega_{k}\left(u_{k}\right)>-\sum_{j \neq k} \omega_{j}\left(u_{k}\right)$, it being observed that the period of the conjugate of $u_{k}$ along $\Gamma_{n+1}$ positively sensed is negative.

It will be convenient to have available the following standard lemma concerning cones in $\boldsymbol{R}^{n}$ generated by vectors of a special kind which subsume the period vectors $\omega(u)$ just considered. For $k=1, \ldots, n$ let $C_{k}=\left(c_{1 k}, \ldots, c_{n k}\right) \in \boldsymbol{R}^{n}$ and have the property that $c_{k k}>0 ; c_{j k}<0, j \neq k ; \sum_{j} c_{j k}>0$. The lemma in question is 
Lemma 3.1. The vectors $C_{1}, \ldots, C_{n}$ form a base for $\boldsymbol{R}^{n}$. The cone generated by them contains the points $x=\left(x_{1}, \ldots, x_{n}\right)$ all of whose coordinates are nonnegative. If not all the $x_{j}$ are zero, the coefficients in the representation $x=\sum \alpha_{k} C_{k}$ are all positive.

For an elementary account of substantially this lemma cf. [6, 136-7].

On introducing the minimal positive harmonic functions on $\Omega$ one obtains with the aid of Lemma 3.1 the following theorem.

Theorem 3.1. Let $\zeta=\left(\zeta_{1}, \ldots, \zeta_{n+1}\right) \in \Gamma_{1} \times \ldots \times \Gamma_{n+1}$. There exists a unique member $f_{\zeta}$ of $P$ having the property that $\operatorname{Re} f_{\zeta}$ vanishes continuously at each point of $\Gamma-\left\{\zeta_{1}, \ldots, \zeta_{n+1}\right\}$.

The proof is very simple. It suffices to introduce $u_{\zeta_{k}}$, the minimal positive harmonic function on $\Omega$ vanishing continuously at each point of $\Gamma-\left\{\zeta_{k}\right\}$ and normalized to satisfy $u_{\zeta_{k}}(a)=1$. If a function $f_{\zeta}$ exists, then $\operatorname{Re} f_{\zeta}=\sum_{1}^{n+1} \mu_{k} u_{\zeta_{k}}$, where $\mu_{k}>0$ and $\sum_{1}^{n+1} \mu_{k}=1$. The $\mu_{k}$ are determined by virtue of Lemma 3.1 as we see on noting that $\sum_{1}^{n+1} \mu_{k} \omega\left(u_{\zeta_{k}}\right)=0$. It follows that there is at most one $f_{\zeta}$. The existence follows from the fact that there is a sequence $\left(\mu_{k}\right)_{1}^{n+1}$ satisfying: $\mu_{k}>0, k=1, \ldots, n+1$; $\sum_{1}^{n+1} \mu_{k}=1 ; \quad \sum_{1}^{n+1} \mu_{k} \omega\left(u_{\zeta_{k}}\right)=0$. The function $\sum_{1}^{n+1} \mu_{k} u_{\zeta_{k}}$ is the real part of an admitted $f_{\zeta}$.

Our concern here is to show

Theorem 3.2. The extreme members of $P$ are precisely the $f_{\zeta}$.

Proof. That each $f_{\zeta}$ is an extreme member of $P$ is easily seen as follows. Suppose that $f_{\zeta}=(1-t) g+t h$, where $g, h \in P$ and $0<t<1$. Then $\operatorname{Re} g=\sum_{1}^{n+1} \alpha_{k} u_{\zeta_{k}}$, where $\alpha_{k} \geqq 0, k=1, \ldots, n+1$, and $\sum_{1}^{n+1} \alpha_{k}=1$. Using the period condition, $\sum_{1}^{n+1} \alpha_{k} \omega\left(u_{\zeta_{k}}\right)=0$, we infer that $\operatorname{Re} g=\operatorname{Re} f_{\zeta}$ and consequently that $g=f_{\zeta}=h$. Hence $f_{\zeta}$ is extreme.

As is to be expected, the main burden of the proof is to show that each extreme member of $P$ is an $f_{\zeta}$. This will be accomplished by showing that if $f$ is an extreme member of $P$, then the set $\left\{\eta \in \Gamma: \lim \sup _{\eta} \operatorname{Re} f>0\right\}$ has at most $n+1$ elements. For the set in question has at least one point on each $\Gamma_{k}$ and so will have as a consequence of the asserted property exactly one point on each $\Gamma_{k}$. We then conclude that $\operatorname{Re} f$ is of the form $\sum_{1}^{n+1} \mu_{k} u_{\zeta_{k}}$ where $\mu_{k}>0$ and $\zeta_{k} \in \Gamma_{k}$ for each $k$, whence $f=f_{\zeta}, \zeta=\left(\zeta_{1}, \ldots, \zeta_{n+1}\right)$.

To establish the asserted boundary property of $f \in P$, we use the following lemma.

Lemma. 3.2. Let $h$ be positive harmonic on $\Omega$ and let $\eta_{1}, \ldots, \eta_{m}$ be $m(\geqq 2)$ distinct points of $\Gamma$ such that $\lim \sup _{\eta_{k}} h>0, k=1, \ldots, m$. Then for given $j \in\{1, \ldots, m\}$ there exists a positive harmonic function $h_{j}$ on $\Omega$ satisfying: $\lim _{\eta} h_{j}=0$ for $\eta \in \Gamma$ near $\eta_{k}$ for each $k \neq j, \lim _{\eta}\left(h-h_{j}\right)=0$ for $\eta \in \Gamma$ near $\eta_{j}$, and $h_{j}<h$. 
Proof. We introduce, as we may, a regular analytic Jordan arc $\alpha$ lying in $\Omega$ save for its endpoints that are on $\Gamma$ such that (1) $\Omega-\alpha$ is not connected, (2) the endpoints of $\alpha$ are also the endpoints of an arc $\beta \subset \Gamma$, such that $\eta_{j}$ is an inner point of $\beta$ and $\eta_{k} \notin \beta, k \neq j$, (3) the component of $\Omega-\alpha$ of which $\eta_{j}$ is a frontier point is the bounded Jordan region $\omega$ satisfying: $\operatorname{fr} \omega=\alpha \cup \beta$.

We introduce $p_{1}$, the smallest positive harmonic function on $\omega$ satisfying the condition that $\lim _{z} p_{1}=h(z), z \in \alpha \cap \Omega$. The function $p_{1}$ has limit 0 at each inner point of $\beta$. We thereupon introduce $S_{1}=p_{1} \cup h \mid(\Omega-\omega)$, which is superharmonic on $\Omega$. We introduce $S_{2}$ analogously, replacing $\omega$ by $\Omega-\bar{\Omega}$. Given a superharmonic function $S$ on a region, not the constant $+\infty$, possessing a harmonic minorant, we let $m S$ denote its greatest harmonic minorant. Since $h<S_{1}+S_{2}$, it follows that $h \leqq m S_{1}+m S_{2}$. From the definition of $S_{1}$ (respectively $S_{2}$ ) we have $m S_{1}, m S_{2}<h$. Taking $h_{j}=m S_{2}$, we see that the requirements of Lemma 3.2 are fulfilled by the $h_{j}$.

Turning to the study of the extreme members of $P$ we suppose that $f$ is an extreme member and that $E=\left\{\eta \in \Gamma: \lim \sup _{\eta} \operatorname{Re} f>0\right\}$ contains at least $n+2$ points. With $h=\operatorname{Re} f$ we introduce $h_{1}, \ldots, h_{n+2}$ of Lemma 3.2 corresponding to distinct points $\eta_{1}, \ldots, \eta_{n+2} \in E$. There exist real numbers $c_{1}, \ldots, c_{n+1}$ where $\max \left|c_{k}\right|=1$ such that $\sum_{1}^{n+1} c_{k} h_{k}$ is the real part of an analytic function $g$ on $\Omega$ satisfying $\operatorname{Im} g(a)=0$. The function $g$ has the property that $\lim _{\eta_{n+2}} \operatorname{Re} g=0$.

Now $\operatorname{Re}[(n+1) f-g]=\sum_{1}^{n+1}\left(h-c_{k} h_{k}\right)$ is positive and less than $(2 n+2) h$. Since $f$ is an extreme member of $P$, we conclude that $(n+1) f-g=c f$ for some positive number $c$. Hence $g=d f$, $d \in \boldsymbol{R}$. Since $\lim _{\eta_{n+2}} \operatorname{Re} g=0$, we conclude that $g$ is the constant 0 . However $\lim \sup _{\eta_{l}}|\operatorname{Re} g|>0$ for some $l \in\{1, \ldots, n+1\}$ as we see by the properties of the $h_{k}$ and the $c_{k}$. Contradiction.

Theorem 3.2 is thereby established.

4. A remark concerning the $f_{\zeta}$. We embed $\Omega$ in a compact Riemann surface $\widetilde{\Omega}$ by Schottky doubling and let $\alpha$ denote the anticonformal involution of $\widetilde{\Omega}$ leaving each point of fr $\Omega$ fixed. Given an allowed $\zeta$, we consider the family $M(\zeta)$ of meromorphic functions on $\widetilde{\Omega}$ having a divisor at least as large as $\delta$ defined as taking the value -1 at each $\zeta_{k}$ and elsewhere the value 0 . We shall see that $\operatorname{dim} M(\zeta)=2$ and $\left\{f_{\zeta}, 1\right\}$ is a generating pair of $M(\zeta)$, it being understood that here $f_{\zeta}$ denotes the meromorphic prolongation to $\widetilde{\Omega}$ of the function $f_{\zeta}$ of Section 3 .

To this end, given $f \in M(\zeta)$, we introduce $g=i(f+\overline{f \circ \alpha})$, which has real part taking the value 0 at each point of fr $\Omega-\left\{\zeta_{1}, \ldots, \zeta_{n+1}\right\}$. Suppose that $g$ is not constant. With $u_{\zeta_{k}}$ denoting here the harmonic prolongation to $\widetilde{\Omega}-\left\{\zeta_{k}\right\}$ of the function so designated in Section 3 we have $\operatorname{Re} g=\sum_{1}^{n+1} \sigma_{k} u_{\zeta_{k}}, \sigma_{k} \in \boldsymbol{R}$. Let $c$ denote a zero of $t \mapsto \min \left\{\mu_{k}-t \sigma_{k}\right\}$, where $\mu_{k}$ is the coefficient entering in the representation of $\operatorname{Re} f_{\zeta}$ of Section 3. (Such a zero exists.) We see that $\operatorname{Re}\left(f_{\zeta}-c g\right)$ is nonnegative in $\Omega$ and its representation as a sum of positive constant multiples of the $u_{\zeta_{k}}$ involves at most $n$ terms. Hence $f_{\zeta}-c g$ is a pure imaginary constant. It follows that $g=$ $A f_{\zeta}+B i, A, B \in \boldsymbol{R}$, without restriction on $g$. A similar result holds with $h=(f-\overline{f \circ \alpha})$ 
replacing $g$. Noting that $2 f=-i g+h$, we conclude the assertion of the preceding paragraph.

5. The case where $S$ is a noncompact Riemann surface having finite topological characteristics and nonpointlike boundary components. We suppose as we may that $S$ is a region of a compact Riemann surface $\tilde{S}$, the frontier of $S$ consisting of a finite number of mutually disjoint regular analytic closed Jordan curves the points of which constitute the fixed point set of an anticonformal involution of $\tilde{S}$ interchanging $S$ and $\tilde{S}-\bar{S}$. Let $g$ denote the genus of $S$; let $c$ denote the number of components of fr $S$; let $\gamma_{1}, \ldots, \gamma_{2 g+(c-1)}$ be representatives of the members of a basis for the 1-dimensional homology group of $S$. Given $u$ harmonic on $S$, let $\omega(u)$ denote the vector in $\boldsymbol{R}^{2 g+(c-1)}$ given by

$$
\omega(u)=\left(\int_{\gamma_{1}} \delta u, \ldots, \int_{\gamma_{2 g+(c-1)}} \delta u\right),
$$

where $\delta u$ is the abelian differential given in terms of a local uniformizer $\theta$ by $-2 i(u \circ \theta)_{z} d z$. The significance of the notation $u_{\xi_{k}}$ of Section 3 is to persist in the present setting.

The following theorem holds.

Theorem 5.1. (1) If $f$ is an extreme member of $P$, then $\operatorname{Re} f$ has limit 0 at each point of $\operatorname{fr} S$ save for at most $2 g+c$ exceptions and consequently is representable as a sum of at most $2 g+c$ minimal positive harmonic functions on $S$.

(2) A member $f$ of $P$ is an extreme member if and only if $\operatorname{Re} f$ admits a representation of the form $\sum_{1}^{m} \mu_{k} u_{\zeta_{k}}$, where $1 \leqq m \leqq 2 g+c, \mu_{k}>0, \sum_{1}^{m} \mu_{k}=1$, the $\zeta_{k}$ are distinct points of $\mathrm{fr} S$, and $\omega\left(u_{\zeta_{j(1)}}\right), \ldots, \omega\left(u_{\zeta_{j(m-1)}}\right)$ are linearly independent for every permutation $j$ of $\{1, \ldots, m\}$ when $m \geqq 2$. The indicated representation of $\operatorname{Re} f$ for extreme $f$ is essentially unique.

(3a) Suppose that $2 g+c \geqq 2$. Given distinct points $\zeta_{1}, \ldots, \zeta_{m} \in \mathrm{fr} S$, there exists an extreme member of $P$ whose meromorphic prolongation to $\tilde{S}$ has poles exactly at the $\zeta_{k}$ if and only if $m \geqq 2$ and $(0, \ldots, 0) \in \boldsymbol{R}^{2 g+(c-1)}$ admits a unique representation as a barycenter of $\omega\left(\mu_{\zeta_{1}}\right), \ldots, \omega\left(u_{\zeta_{m}}\right)$ with positive masses. There is at most one such member. The case, $m=2$, occurs for all $(g, c)$ with $g \geqq 1, c=1$ or 2 .

(3b) Suppose that $2 g+c=1$. Then (3a) holds with the conditional clause replaced by "if and only if $m=1$ ".

Proof. (1) When $2 g+c \geqq 2$, the corresponding developments of Section 3 may be paraphrased. Here a 1-dimensional homology basis has $2 g+(c-1)$ elements. The case, $2 g+c=1$, follows from Section 2 .

(2) Using (1) we see that if $f$ is an extreme member of $P$, then $\operatorname{Re} f$ admits a representation of the stated form apart from the asserted linear independence of $\omega\left(u_{\zeta_{j(1)}}\right), \ldots, \omega\left(u_{\zeta_{j(m-1)}}\right)$.

If the vectors in question were linearly dependent, there would exist $h$ analytic 
on $S$ satisfying: $\operatorname{Re} h=\sum_{1}^{m-1} \lambda_{k} u_{\zeta_{j(k)}}$, where $\lambda_{k} \in \boldsymbol{R}$ and $\max \lambda_{k}=1$ and $\operatorname{Im} h(a)=0$. Let $\alpha=\min \left\{\lambda_{k}^{-1} \mu_{j(k)}: \lambda_{k}>0\right\}$. We see that $\operatorname{Re}(f-\alpha h)$ would be a sum of the form $\left(\sum_{1}^{m-1} v_{k} u_{\zeta_{j(k)}}\right)+\mu_{j(m)} u_{\xi_{(m)}}$, where min $v_{k}=0$. Since $f$ is extreme, $h$ would be a constant multiple of $f$. The factor in question would be positive since $\max \lambda_{k}=1$ implies that $\operatorname{Re} h$ is not bounded above. We conclude that $\mu_{j(m)}=0$. Contradiction. The asserted linear independence follows.

Conversely, suppose that $f \in P$ satisfies the condition stated in (2). Suppose that $f=(1-t) f_{1}+t f_{2}$ where $f_{1}, f_{2} \in P$ and $t \in(0,1)$. Then $\operatorname{Re} f_{j}=\sum_{k=1}^{m} \sigma_{k, j} u_{\zeta_{k}}$, $j=1,2$, where $\sigma_{k, j} \geqq 0$ and $\sum_{k=1}^{m} \sigma_{k, j}=1$. We conclude that $f_{1}=f_{2}$, the period condition being used when $m \geqq 2$. Hence $f$ is an extreme member of $P$.

The second assertion of (2) is immediate.

Sufficiency (3a), (3b). Each case is immediate.

Necessity (3a). Let $f$ be an extreme member of $P$ of the stated kind, so that $m \geqq 2$ (otherwise $g=0$ and $c=1$ ) and $\operatorname{Re} f=\sum_{1}^{m} \mu_{k} u_{\zeta_{k}}$, where $\mu_{k}>0$ and $\sum_{1}^{m} \mu_{k}=1$. If the stated barycenter condition did not hold, there would exist $\lambda_{k} \in \boldsymbol{R}, k=1, \ldots, m$, satisfying: $\max \lambda_{k}>0, \sum_{1}^{m} \lambda_{k}=0, \sum_{1}^{m} \lambda_{k} \omega\left(u_{\zeta_{k}}\right)=0$. Consequently, there would exist $h$ analytic on $S$ satisfying: $\operatorname{Re} h=\sum_{1}^{m} \lambda_{k} u_{\zeta_{k}}, h(a)=0$. Since $f$ is an extreme member of $P$, for $\alpha$ and $\beta$ small and positive $\alpha(f+\beta h)$ would be a constant multiple of $f$, and so $h$ would be also. Since $h(a)=0, h$ would be the constant 0 . This is not the case. Necessity follows.

Necessity (3b). The discussion is routine and will be omitted.

The uniqueness statements are immediate.

The observation concerning the realization of the case, $m=2$, is vacuous for (3b) and is established for (3a) by considering $S$ admitting a 2-sheeted covering of the open unit disk with $2 g+\mathrm{c}$ distinct ramification points.

6. Remark. The observations of Section 4 persist, mutatis mutandis, for the extreme functions considered in Section 5.

\section{References}

[1] Ahlfors, L. V.: Open Riemann surfaces and extremal problems on compact subregions. Comment. Math. Helv. 24, 1950, 100-134.

[2] Courant, R.: Dirichlet's principle, conformal mapping, and minimal surfaces, with an appendix by M. Schiffer. - Interscience Publishers, Inc., New York-London, 1950.

[3] Forelli, F.: The extreme points of some classes of holomorphic functions. - Duke Math. J. 46, 1979, 763-772.

[4] Garabedian, P. R.: Schwarz's lemma and the Szegö kernel function. - Trans. Amer. Math. Soc. $67,1949,1-35$.

[5] GRUNSKY, H.: Eindeutige beschränkte Funktionen in mehrfach zusammenhängenden Gebieten. I. - Jber. Deutsch. Math. Verein. 50, 1940, 230-255. II. - Ibid. 52, 1942, 118-132.

[6] GRUNSKY, H.: Lectures on theory of functions in multiply connected domains. - Studia Math. Skript 4, Vandenhoeck \& Ruprecht, Göttingen, 1978. 
[7] Heins, M.: A lemma on positive harmonic functions. - Ann. of Math. 52, 1950, 568-73.

[8] Heins, M.: Carathéodory bodies. - Ann. Acad. Sci. Fenn. Ser. A I Math. 2, 1976, 203-232.

[9] Kelley, J. L., I. NamiokA, and co-authors: Linear topological spaces. - D. Van Nostrand Company, Inc., Princeton, New Jersey-Toronto-New York-London, 1963.

[10] Myrberg, P. J.: Über die analytische Fortsetzung von beschränkten Funktionen. - Ann. Acad. Sci. Fenn. Ser. A I Math. - Phys. 58, 1949, 1-7.

[11] RiemanN, B.: Bernhard Riemann's gesammelte mathematische Werke und wissenschaftlicher Nachlass. - Herausgegeben von H. Weber, Druck und Verlag von B. G. Teubner, Leipzig, 1892. Reprint. Dover Publications, New York, 1953.

University of Maryland

Department of Mathematics

College Park, Maryland 20742

USA

Received 7 October 1983 